\title{
Real-time monitoring of a coffee roasting process with near infrared spectroscopy using multivariate statistical analysis: A feasibility study
}

\author{
Tiago A. Catelani ${ }^{\mathrm{a}}$, João Rodrigo Santos ${ }^{\mathrm{b}}$, Ricardo N.M.J. Páscoa ${ }^{\mathrm{c}, *}$, Leonardo Pezza ${ }^{\mathrm{a}}$, \\ Helena R. Pezza ${ }^{a}$, João A. Lopes ${ }^{\mathrm{d}}$ \\ a Instituto de Química, Universidade Estadual Paulista “Julio de Mesquita Filho", UNESP, R. Prof. Francisco Degni 55, P.O. Box 355, 14800-900 Araraquara, SP, Brazil \\ ${ }^{\mathrm{b}}$ LAQV/REQUIMTE - Departamento de Química e Bioquímica, Faculdade de Ciências, Universidade do Porto, Porto, Portugal \\ ${ }^{\mathrm{c}}$ LAQV/REQUIMTE, Laboratório de Química Aplicada, Departamento de Ciências Químicas, Faculdade de Farmácia, Universidade do Porto, Porto, Portugal \\ ${ }^{\mathrm{d}}$ Research Institute for Medicines (iMed.ULisboa), Faculdade de Farmácia, Universidade de Lisboa, Lisboa, Portugal
}

\section{A R T I C L E I N F O}

\section{Keywords:}

Coffee roasting

Real-time monitoring

Multivariate statistical process control

Near-infrared spectroscopy

Principal component analysis

\begin{abstract}
A B S T R A C T
This work proposes the use of near infrared (NIR) spectroscopy in diffuse reflectance mode and multivariate statistical process control (MSPC) based on principal component analysis (PCA) for real-time monitoring of the coffee roasting process. The main objective was the development of a MSPC methodology able to early detect disturbances to the roasting process resourcing to real-time acquisition of NIR spectra. A total of fifteen roasting batches were defined according to an experimental design to develop the MSPC models. This methodology was tested on a set of five batches where disturbances of different nature were imposed to simulate real faulty situations. Some of these batches were used to optimize the model while the remaining was used to test the methodology. A modelling strategy based on a time sliding window provided the best results in terms of distinguishing batches with and without disturbances, resourcing to typical MSPC charts: Hotelling's $\mathrm{T}^{2}$ and squared predicted error statistics. A PCA model encompassing a time window of four minutes with three principal components was able to efficiently detect all disturbances assayed. NIR spectroscopy combined with the MSPC approach proved to be an adequate auxiliary tool for coffee roasters to detect faults in a conventional roasting process in real-time.
\end{abstract}

\section{Introduction}

Coffee is one of the most appreciated and consumed beverages around the world. The global coffee production in 2015 was estimated by the International Coffee Organization as 143.4 million tons of coffee bags $(60 \mathrm{~kg}$ ) [1]. The demand of coffee is increasing and it is expected to continue in the near future propelled by its consumption in emerging markets as Russia, Australia, South Korea, and others [1]. Therefore, coffee roasters have been continuously promoting activities to increase the quality standards of roasted coffee. This requires a deep knowledge of the characteristics of green coffee beans/origins as well as upon the roasting process. However, it is difficult to control the characteristics of green coffee beans due to the inherent crop-to-crop variability. The development of analytical methodologies for the in-line monitoring of the roasting process is a major step forward for coffee roasters to better understand the coffee roasting process on the production environment [2]. The roasting process may be performed according under three strategies [3]: 1) conventional - raw coffee is poured inside horizontal or vertical drums and motion of the beans is performed by paddles or through rotation of the drum; 2) fluidized beds - an upward hot stream of air assures roasting and motion of the beans and 3) fast roasting faster heat transfer is achieved through increased hot streams of air enabling the roasting process to be concluded within 2-4 min. The final organoleptic characteristics of the roasted coffee (aroma, taste, colour, flavour) and of the coffee beverage are known to be highly dependent on the roasting process [4].

The application of NIR spectroscopy in monitoring processes is wide and broad $[2,5,6]$ but only recently has been applied for the real-time monitoring of the coffee roasting process $[2,7]$. The major advantages of this technique in monitoring processes are the possibility for in-line and real time analysis without sampling and/or sample preparation. The coffee roasting process control usually rely on experts that through the combination of visual, olfactory and/or acoustic properties of coffee beans are able to select the roasting degree [8,9]. However, this control is not very effective because process disturbances are not or only detected at the end. Thus, exploiting NIR spectroscopy and multivariate

\footnotetext{
* Corresponding author.

E-mail address: rnpascoa@ff.up.pt (R.N.M.J. Páscoa).
} 
statistical process control (MSPC) based on latent variable (LV) models such as principal component analysis (PCA), may be an interesting alternative tool to control the roasting process.

The roasting process can be considered a dynamic batch process [10]. For that reason, MSPC can be applied to monitor this process because it is able to detect changes in the covariance structure of the process variables [11]. A typical method development relies in a model calibration with data collected from processes operated under normal conditions. These conditions ensure that the product is within its quality specifications [10]. Ideally, roasted coffee batches should be produced by regulatory operating conditions within the design-space for that particular product [11]. Experiments needed to calibrate the MSPC model should be carried out within the design-space limits, to cover the normal operating conditions. Deviations from normal process behaviour are detected when changes on covariance matrix occur or abnormal signal components arise. The goal is to identify process disturbances as soon as they occur [10]. The most common causes of disturbances in batch processes involve sensors/equipment failure and the presence of impurities or defects in the raw materials $[10,11]$. Failure upon reproducing pre-established experimental roasting conditions, namely, the initial mass weighing and/or selection of the raw material (green coffee beans) composition (species and varieties) and/ or time-temperature heating profile may be considered the most probable causes of faulty roasting processes in automated coffee roaster facilities $[10,11]$. An incorrect blend composition and/or control of the roasting process markedly change the organoleptic profile of the brewed coffee. In MSPC-PCA based models, Hotelling's $\mathrm{T}^{2}$ and squared prediction error $(Q)$ are the most commonly used statistics $[10,11]$. Recent works that used these models coupled with NIR spectroscopy have been applied on the monitoring of traditional Chinese medicine production [12] and detection of adulterations in wheat products [13]. The results obtained revealed a good accuracy.

Regarding real-time monitoring of coffee roasting process, there are few approaches described in the literature $[2,7,9,14-17]$ and non-exploits the use of MSPC coupled with NIR spectroscopy. Some the existent works in this area measure the transient chemical signature of volatile organic compounds (VOCs) present in process gases formed during coffee roasting by proton transfer reaction time-of-flight mass spectrometry (PTR-ToF-MS) [14-16]. These compounds are important for the final flavour of coffee but the absence of some of these compounds cannot be used as indicators of a good roasting process since its formation depends on the raw material and roasting conditions. Moreover, the analytical PTR-ToF-MS equipment's cost is a major drawback. Another interesting approach exploits the use of cracking sounds emitted by coffee beans during the roasting process. However, it is a preliminary work performed with only one coffee sample [9]. Electron paramagnetic resonance (EPR) spectroscopy was also used to quantify free radicals in Arabica and Robusta varieties. This work brings more knowledge about the dynamics of free radicals generation in the roasting process but it is not suitable for monitoring the whole process [17]. Regarding NIR technology, it should be referred that the real-time monitoring of acidity, coffee colour and sucrose content as well as the antioxidant properties in the roasting process was already performed $[2,7,18]$. This work proposes the use of NIR spectroscopy and multivariate data analysis to monitor the coffee roasting process, allowing for the early identification of deviations from the targeted product characteristics.

\section{Materials and methods}

\subsection{Batch roasting procedure}

Coffee batches were roasted using a Hottop Coffee roaster KN$8828 \mathrm{~B}-2 \mathrm{~K}$. This is a pilot-scale equipment designed to roast coffee in a traditional fashion. The equipment presents a roasting drum (where the green coffee beans are poured) that rotates continuously, during the roasting process, upon an horizontal axis. This equipment enables the roasting process to be controlled in terms of heating power and fan speed. The roasting temperature was studied by selecting different values of the roasting power and the fan speed was maintained constant (at 25\%) throughout the batch (always the same setting for all batches) with the objective of not interfering with the roasting temperature besides the heating power. Furthermore, the coffee roaster features a frontal glass window that enables direct visualization of the roasting drum, through which NIR signal was acquired. More details can be found in [7].

The coffee beans were purchased from a local seller and included both Arabica (from Brazil and Timor) and Robusta species (from Uganda). The nominal roasting conditions were established according to the equipment manufacturer recommendations for typical roasting process (in terms of mass, roasting power and fan speed), see Table 1 (batches 1-15). Under these experimental conditions, each coffee batch

Table 1

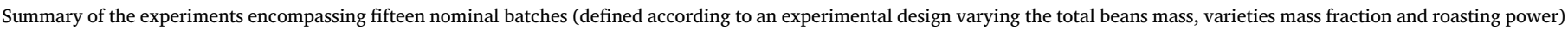
and five batches where different disturbances were imposed.

\begin{tabular}{|c|c|c|c|c|c|c|c|}
\hline \multirow[t]{2}{*}{ Batch ID } & \multirow[t]{2}{*}{ Total mass $(\mathrm{g})$} & \multicolumn{3}{|c|}{ Mass fraction (\%) } & \multirow[t]{2}{*}{ Roasting power } & \multirow[t]{2}{*}{ Batch type } & \multirow[t]{2}{*}{ Data set } \\
\hline & & Arabica Brazil & Arabica Timor & Robusta Uganda & & & \\
\hline B1 & 245.0 & 95 & 5 & 0 & 5 & Nominal & Testing \\
\hline B2 & 245.0 & 100 & 0 & 0 & 5 & Nominal & Validation \\
\hline B3 & 245.0 & 95 & 0 & 5 & 5 & Nominal & Calibration \\
\hline B4 & 247.5 & 95 & 5 & 0 & 5 & Nominal & Testing \\
\hline B5 & 247.5 & 100 & 0 & 0 & 5 & Nominal & Validation \\
\hline B6 & 247.5 & 95 & 0 & 5 & 5 & Nominal & Calibration \\
\hline B7 & 250.0 & 95 & 5 & 0 & 5 & Nominal & Calibration \\
\hline B8 & 250.0 & 100 & 0 & 0 & 5 & Nominal & Calibration \\
\hline B9 & 250.0 & 95 & 0 & 5 & 5 & Nominal & Calibration \\
\hline B10 & 252.5 & 95 & 5 & 0 & 5 & Nominal & Calibration \\
\hline B11 & 252.5 & 100 & 0 & 0 & 5 & Nominal & Calibration \\
\hline B12 & 252.5 & 95 & 0 & 5 & 5 & Nominal & Removed \\
\hline B13 & 255.0 & 95 & 5 & 0 & 5 & Nominal & Removed \\
\hline B14 & 255.0 & 100 & 0 & 0 & 5 & Nominal & Calibration \\
\hline B15 & 255.0 & 95 & 0 & 5 & 5 & Nominal & Removed \\
\hline B16 & 250.0 & 70 & 0 & 30 & 5 & Non-nominal & Testing \\
\hline B17 & 250.0 & 100 & 0 & 0 & 6 & Non-nominal & Validation \\
\hline B18 & 250.0 & 100 & 0 & 0 & 4 & Non-nominal & Testing \\
\hline B19 & 220.0 & 100 & 0 & 0 & 5 & Non-nominal & Validation \\
\hline B20 & 280.0 & 100 & 0 & 0 & 5 & Non-nominal & Validation \\
\hline
\end{tabular}


was roasted for approximately $24 \mathrm{~min}$ in which the temperature increased from $75{ }^{\circ} \mathrm{C}$ to approximately $210{ }^{\circ} \mathrm{C}$. These roasting conditions, similar to conventional process roasting conditions, provided a coffee of medium roast intensity. Variations around a $250 \mathrm{~g}$ batch made of $100 \%$ Arabica Brazil were imposed according to a D-optimal design with no central points or replicates. The design factors were residuals amounts of Arabica (of a different provenience) and Robusta and the total amount of beans (initial mass). These variations were imposed to simulate the natural variability of a roasting batch as follows: the total batch mass was varied between 245 and $255 \mathrm{~g}$. Batches including $5 \%$ of an additional specie (Robusta/Uganda) and provenience (Arabica/ Timor) were also produced. These batches were designated as nominal batches (NOC) and the imposed variations were considered to be acceptable for the production of roasted coffee with similar final characteristics.

Five batches with imposed disturbances (or non-nominal batches) were produced to simulate abnormal roasting conditions and to verify the detection ability of the MSPC method. As aforementioned, the most common failures of a coffee roasting industrial process are related with mass weighing, roasting power adjustment and raw-material (green coffee beans) composition (Robusta/Arabica). Therefore, the nonnominal batches included minor deviations from the aforementioned reference conditions for NOC. Batch 16 used a different raw-material blend (higher amount of Robusta/Uganda beans). Batches 17 and 18 were roasted considering a higher and lower roasting power, respectively. Batches 19 and 20 used a lower and higher initial mass of green beans, respectively (Table 1). Although these five non-nominal batches do not cover all sources of possible faults (and severity), they encompass most of the main plausible sources of coffee roasting process errors.

\subsection{In-line process monitoring}

The in-line roasting process monitoring was performed using a Fourier transform near-infrared spectrometer (FTLA 2000, ABB, Quebéc, Canada) featuring an indium-gallium-arsenide (InGaAs) detector and controlled via Bomen-Grams software (version 7, ABB, Quebéc, Canada). The near-infrared spectra were acquired through a stainless steel diffuse reflectance probe (SabIR, ThermoNicolet, Madison, Wisconsin), featuring a sapphire window of $1.0 \mathrm{~cm}$ diameter and $0.2 \mathrm{~cm}^{2}$ illumination area. The probe was placed outside the coffee roaster at approximately $2 \mathrm{~mm}$ distance of the frontal glass window. Each acquired spectrum resulted from an average of 64 scans with a resolution of $8 \mathrm{~cm}^{-1}$ over a wavenumber interval from 10,000 to $4000 \mathrm{~cm}^{-1}$. A background was made at the beginning of each roasting procedure using a PTFE disk (Teflon). A total of 24 spectra were collected (one spectrum per minute), thus a total of 480 spectra considering the twenty batches produced. Raw spectra were preprocessed with standard normal variate (SNV) followed by Savitzky-Golay (19 points filter width and second-order polynomial and first derivative) to correct for scale and baseline variations. Additional details regarding the experimental setup can be found in [2].

\subsection{Multivariate data analysis}

Batch spectral data were initially arranged as a three-way array $X$ ( $I$ $\mathrm{x} J \times K$ ), where $I$ is the index for batch, $J$ for time and $K$ for wavenumber. This array was unfolded preserving the wavenumbers mode yielding a $I K \times J$ array [19]. The MSPC methodology was performed based on principal component analysis (PCA) models [20]. To better capture the roasting process evolution, time slices of different sizes were considered (here called the sliding window strategy) [21,22]. The methodology starts by defining a time slice size (sliding window size, WS). The original $I K \times J$ array is then split into several matrices each containing process data considering each slice of WS size and a PCA model is fitted to each data slice. The first model uses spectra acquired from 1 to WS minutes. A second model will consider spectra from 2 to WS $+1 \mathrm{~min}$ and so on, until the end of the batch. Consequently, when the WS is set to 24 (the entire duration of the batch) the result is a single PCA model covering the entire batch duration. All PCA models were calibrated with mean centred data. The control charts produced in this work resourced to the squared prediction error $(Q)$ and Hotelling's $T^{2}$ statistics [11]. Confidence limits for both statistics were calculated according to Nomikos and Macgregor [23,24]. Control charts are represented in terms of the reduced values of Hotelling's $\mathrm{T}^{2}$ and $\mathrm{Q}$ obtained, dividing each statistic by the corresponding $95 \%$ confidence limit. Therefore, the control limit for 95\% confidence level was 1 in both charts. Chemometric models were developed with Matlab version 8.3 (MathWorks, Natick, MA, USA) and the PLS Toolbox version 7.5 (Eigenvector Research Inc., Wenatchee, WA, USA).

\section{Results and discussion}

\subsection{Spectra analysis}

A total of twenty roasting batches (Table 1 ) were monitored using FT-NIR spectroscopy, resulting on a three-way array X (20 batches, 24 points per batch and 1556 variables). After unfolding, a two-dimensional array (480 rows resulting from 20 batches $\times 24$ points and 1556 columns) preserving the wavelength direction was obtained. Typical spectra (batch B1) obtained under NOC conditions are illustrated in Fig. 1. The gradual reduction of intensity in the water-related bands is clear and is related with the decrease of moisture content during the roasting process. To facilitate the analysis, spectra were divided in five different regions according to previous knowledge [2]: $9800-7661 \mathrm{~cm}^{-1}$ (R1), 7660-6120 cm $\mathrm{cm}^{-1}$ (R2), 6119-5501 $\mathrm{cm}^{-1}$ (R3), $5500-4961 \mathrm{~cm}^{-1}$ (R4) and $4960-4150 \mathrm{~cm}^{-1}$ (R5). Spectral bands that undergo major variations are located between R2 and R5. However, based on a previous work [2], the best spectral regions for monitoring the sucrose content and beans colour were R3 and R4. Moreover, several important compounds that are related with the quality of roasted coffee are spectrally active in these regions. Therefore, further analysis was restricted to regions R3 and R4 $\left(6120-4961 \mathrm{~cm}^{-1}\right)$ in order to incorporate the major chemical changes observed in the NIR spectra. Within this region, the variation related with moisture decrease around $5200 \mathrm{~cm}^{-1}$ was preserved. This region is within the first overtone region and part of the combination band region. It captures $\mathrm{C}-\mathrm{H}$ and $\mathrm{S}-\mathrm{H}$ vibrations in the first overtone region and $\mathrm{C}=\mathrm{O}$ plus $\mathrm{O}-\mathrm{H}$ and $\mathrm{O}-\mathrm{H}$ vibrations in the combination band region. The band assignments (Table 2) on the spectral region between 6120 and $4961 \mathrm{~cm}^{-1}$ suggest its suitability to monitor and control the roasting process.

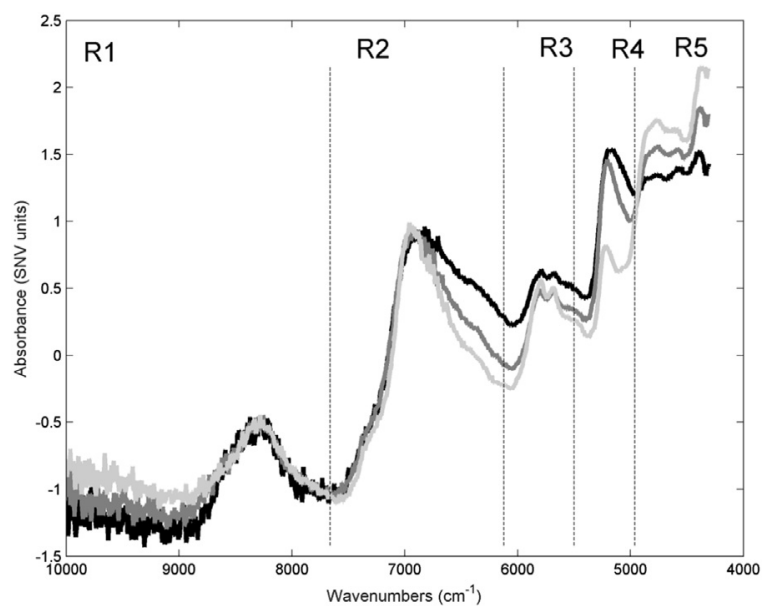

Fig. 1. SNV corrected NIR spectra acquired from batch 1 at different roasting periods (black: $0 \mathrm{~min}$, grey: $12 \mathrm{~min}$; light-grey: $24 \mathrm{~min}$ ). 
Table 2

Band assignments for the spectral regions that more contributed for the principal components loadings.

\begin{tabular}{|c|c|c|c|c|}
\hline Spectral region $\left(\mathrm{cm}^{-1}\right)$ & {$[23]$} & {$[24]$} & [25] & {$[26]$} \\
\hline $5149-4960$ & Chlorogenic acid, water, protein, sucrose & Protein & Carbohydrates & Caffeine \\
\hline $5061-5020$ & Protein & Protein & Carbohydrates & Caffeine \\
\hline $5211-5171$ & Water & & Carbohydrates, lipids, caffeine, proteins, water, chlorogenic acid & \\
\hline $5230-5189$ & Carboxylic acids, caffeine & & Carbohydrates, lipids, caffeine, proteins, water, chlorogenic acid & \\
\hline $5269-5230$ & Chlorogenic acid & & & \\
\hline 5299 & & & & Caffeine \\
\hline 5328 & & Carbohydrates & & \\
\hline $5459-5420$ & Carbohydrates & Carbohydrates & & \\
\hline $5519-5420$ & & Carbohydrates & & \\
\hline $6024-5924$ & & Lipids & Caffeine, trigonelline & Caffeine \\
\hline
\end{tabular}

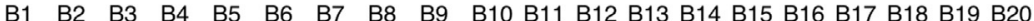
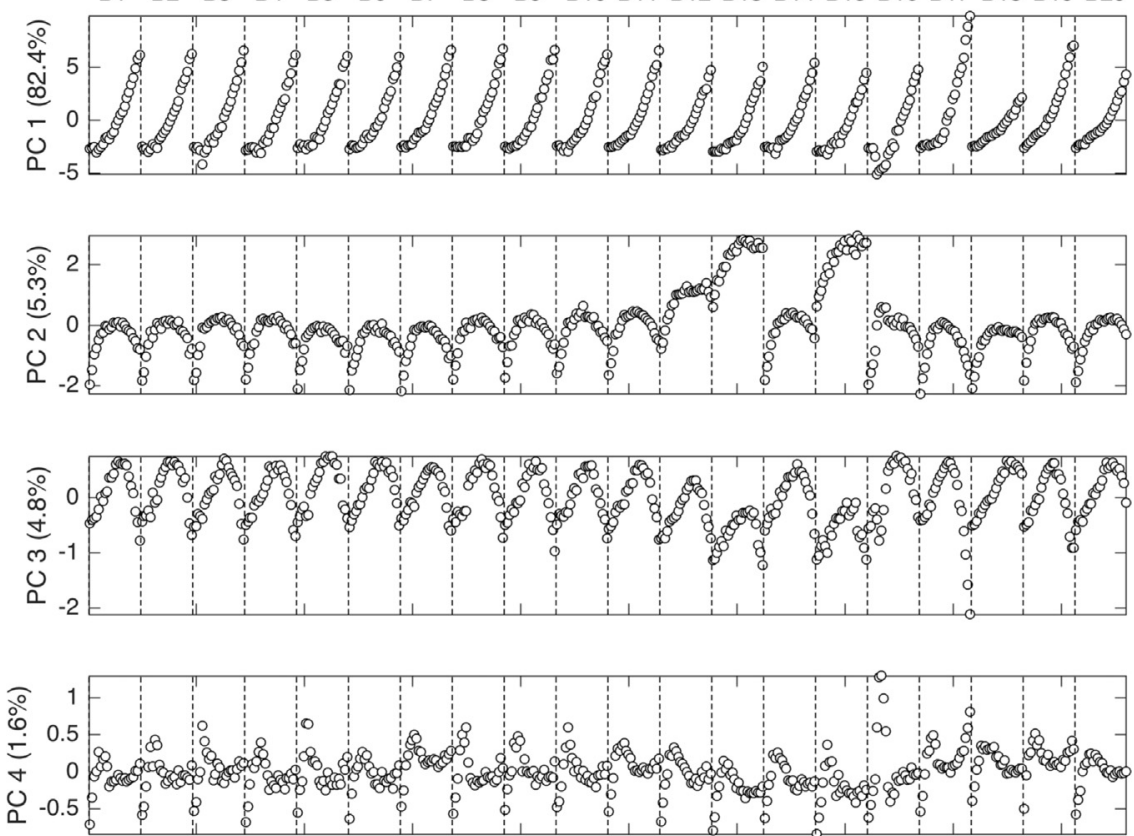

Fig. 2. Scores obtained from a PCA model considering mean centred preprocessed NIR spectra (SNV and Savitzky-Golay with first derivative) of the twenty batches considering the spectral region $6120-4961 \mathrm{~cm}^{-1}$.

\subsection{Exploratory data analysis}

A preliminary PCA model considering the $6120-4961 \mathrm{~cm}^{-1}$ region was built using all batches (from time $0-24 \mathrm{~min}$ ) with the objective of screening the differences between spectral profiles (Fig. 2) between NOC and non-nominal batches. Therefore, each batch (B1, B2, etc) is represented by 24 time points (scores). The scores' variations reflect the spectral changes originating from green coffee beans during the roasting process.

Analysing the scores obtained in PC1, batches B16, B17, B18, B19 and $\mathrm{B} 20$ revealed some differences when compared to NOC batches. The reasons for the differences observed in the scores of PC1 of B16 are related with the high proportion of Robusta coffee beans. Considering the compounds that could have a NIR signal in this spectral region and also the different composition between Arabica and Robusta green coffee beans, the main compounds responsible for these differences may be caffeine, trigonelline and the lipids content as reported by [25]. For batches B17 and B19, the scores were substantially higher at the end of the process, probably due to a darker colour of the roasted coffee beans in these two batches justified by the experimental conditions used in both batches. Batch B17 used more roasting power and B19 used a lower amount of raw material. Although the imposed variations are of different nature, it makes sense that the roasted coffee colour was similar. Regarding batches B18 and B20 (and in accordance with the comments made for B17 and B19), the differences may be justified by the lighter colour of the roasted beans. The most significant wavenumbers of PC1 loadings (data not shown) were located in the $5150-4960 \mathrm{~cm}^{-1}$ and $6020-5925 \mathrm{~cm}^{-1}$ regions. These regions have been correlated to several compounds namely, chlorogenic acid [26], water [26], protein [26,27], carbohydrates [26,28], caffeine [29] and caffeine [28,29], trigonelline [28], lipids [27], respectively.

Concerning the PC2 (Fig. 2), only batches B17 and B18 are slightly different. Batch B17 has lower values at the end of the process and batch B18 has higher values at the end of the process. However, it should be referred that the NOC batches B12, B13 and B15 are also different. The scores of these batches are always higher indicating that some problem occurred in these batches. The wavenumbers that contributed more for PC2 loadings (data not shown) are located in the regions $5270-5230 \mathrm{~cm}^{-1}, 5060-5020 \mathrm{~cm}^{-1}, 5460-5420 \mathrm{~cm}^{-1}$ and at $5345 \mathrm{~cm}^{-1}$. The first two regions may be associated with the content of chlorogenic acids [26] and protein [26,27], carbohydrates, caffeine [29], respectively. The wavenumbers in the range of 5460-5420 $\mathrm{cm}^{-1}$ and around $5345 \mathrm{~cm}^{-1}$ can be associated with the presence of carbohydrates $[26,27]$. The differences found in the PC2 scores of batches B12, B13, B15, B17 and B18 are related with these chemical compounds but the authors did not find any explanation for this pattern. In PC3 (Fig. 2), batches B17 and B18 are again different. Batch B18 follows a different trajectory. It was also visible that the NOC batches B13 and B15 presented lower scores at the beginning of the process. The most important wavenumbers in the PC3 loadings (data not shown) were found at $5230-5190 \mathrm{~cm}^{-1}$ and $5345 \mathrm{~cm}^{-1}$. The range between 5230 and $5190 \mathrm{~cm}^{-1}$ can be associated with the presence of carboxylic acids 
[26], caffeine [26,28], carbohydrates, lipids, proteins and water [28] while carbohydrates [27] can be spectrally active around $5345 \mathrm{~cm}^{-1}$. The differences found in PC3 scores for batches B17 and B18 can be attributed, again, to the different colour development of the coffee beans along the roasting process. The chemical compounds assigned to these wavenumbers were the same used for modelling the roasted beans colour [2]. The reasons why batches B13 and B15 presented different scores are not clear but might be due to the experimental conditions (e.g., a different angle/distance between the probe tip and the roaster glass window inducing an abnormal spectral pattern). In PC4 (Fig. 2), the only batch that differs significantly batch B16. During the roasting process it presented higher scores. The wavenumbers with the highest contributions for the PC4 loadings (data not shown) were found in the ranges of $5210-5170 \mathrm{~cm}^{-1}, 5460$ to $5420 \mathrm{~cm}^{-1}$ and at $5300 \mathrm{~cm}^{-1}$. The wavenumbers between 5460 and $5420 \mathrm{~cm}^{-1}$ and $5210-5170 \mathrm{~cm}^{-1}$ can be associated with the carbohydrates content $[26,27]$ and water $[26,28]$, carbohydrates, lipids, caffeine, chlorogenic acid, proteins [28], respectively. The wavenumbers around $5300 \mathrm{~cm}^{-1}$ can be attributed to the caffeine content [29]. The explanation for the differences found in PC4 scores of batch B16 could be the same referred for PC1.

Regarding the NOC batches a similar pattern was expected since only minor variations were imposed. However, NIR spectra captured a different pattern in some batches (specifically B12, B13 and B15, all NOC) containing some unexpected spectral variability within the $6500-4500 \mathrm{~cm}^{-1}$ region. As this behaviour cannot be considered normal it was decided to remove these experimental data from further analysis.

\subsection{MSPC methodology}

\subsubsection{MSPC methodology using the entire roasting process}

All PCA models for the MSPC strategy were developed on a fixed set of NOC batches, designated as the calibration batches: B3, B6-11, B14. Models optimization required a validation set, composed by NOC (B2 and B5) and abnormal batches (B17, B19 and B20). The validation batches were exclusively used to optimize the MSPC strategy in terms of the best number of principal components. A third set, encompassing NOC and abnormal batches, was defined to externally test the strategy and evaluate the optimised model (unseen data): B1, B4, B16, B18. The division of the batches in calibration, validation and testing sets was randomly performed (see Table 1).

A PCA model with two components was calibrated using only the calibration batches (see Table 1) and the selected spectral region $\left(6120-4960 \mathrm{~cm}^{-1}\right)$. For this model two components encompassed approximately $97 \%$ of the total variance. The validation and testing batches were then projected (Fig. 3). A first observation of the score plot revealed that the scores are not normally distributed and therefore the confidence limits (95\%) are not valid here. Nevertheless, some batches have a different pattern when compared with the grey area (defined by the calibration batches' scores). Specifically, batches B16 and B17 (both abnormal batches) are notoriously distinct from the remaining. In this model, it was not possible to clearly verify different patterns in the remaining abnormal batches (B18, B19 and B20), at least when analysing the scores and consequently in the Hotelling's $\mathrm{T}^{2}$ statistic (the model residuals may have been affected). Additionally, the score plot showed that using a PCA model considering the entire process time range (WS $=24 \mathrm{~min}$ ) is not adequate to model all changes that are occurring in the coffee beans over time. Therefore, using a single model to describe the entire process may not be adequate to identify and detect process disturbances, despite the fact that for some batches the disturbances were identified. These results indicate that the development of time local models may be more adequate for the coffee roasting process monitoring due to some sort of non-linear behaviour that could be happening in the roasting process. Therefore, it is recommended to evaluate if the sliding window strategy may provide a better fault detection ability.

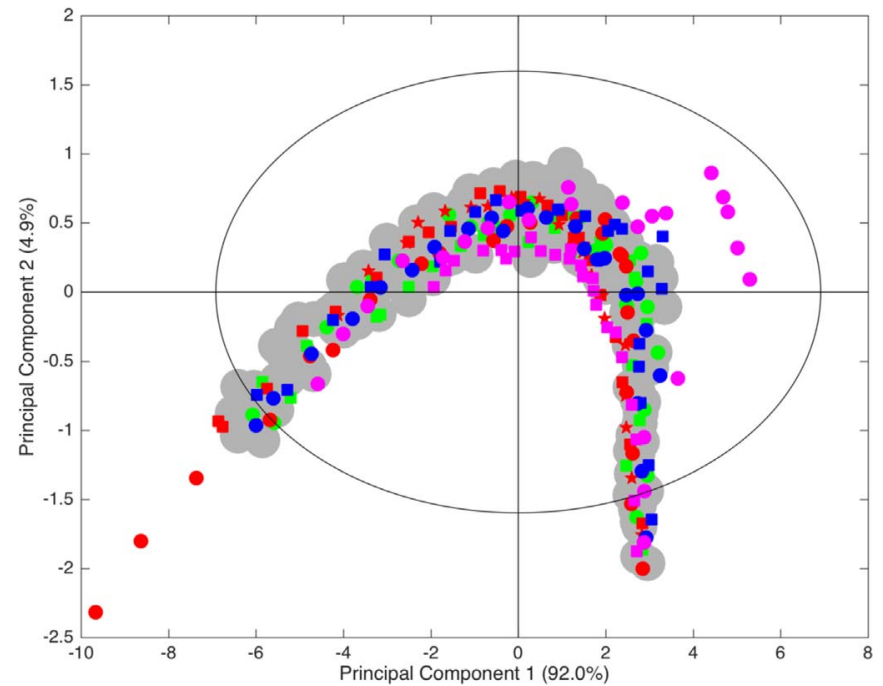

Fig. 3. Score plot from a PCA model developed with calibration batches $(\bigcirc 3,6,7,8,9$, $10,11,14)$ considering the spectra range of $6120-4961 \mathrm{~cm}^{-1}$ and WS $=24 \mathrm{~min}$ and the

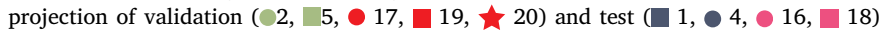
batches. The boundary defines the $95 \%$ confidence limit considering normally distributed scores.

\subsubsection{Optimization of the MSPC methodology using the time sliding window} strategy

The more appropriated sliding window size (WS) and the number of principal components was firstly evaluated using the validation set. The size of the time window was varied from 1 to $24 \mathrm{~min}$. A $24 \mathrm{~min}$ time window (WS $=24 \mathrm{~min}$ ) corresponds to a situation where only one model is created. The number of principal components (PC) tested ranged between 1 and 8 . All the combinations of these factors (24 time windows $\times 8$ components) were tested. The optimization of the MSPC time sliding window modelling strategy was based on the ability of the method to detect deviations in abnormal batches (statistics above the 95\% confidence limit (1) in both charts) while signalling a normal behaviour for NOC batches (statistics below the 95\% confidence limit (1) in both charts). In other words, maximizing the sensitivity and specificity or minimizing the number of false positives and false negatives. Thus, it was considered that all measurements in abnormal batches should yield an out-of-control signal while all measurements in NOC batches should yield a controlled situation. To facilitate the identification of the best combination of PC's and WS, a statistic $D$ was estimated for each possible combination, according to Eq. (1).

$D_{P C, W S}=\alpha\left(Q_{P C, W S}^{V A L-A B N}-Q_{P C, W S}^{V A L-N O C}\right)+(1-\alpha)\left(T_{P C, W S}^{V A L-A B N}-T_{P C, W S}^{V A L-N O C}\right)$

In Eq. (1), $\mathrm{Q}^{\mathrm{VAL}-\mathrm{ABN}}$ and $\mathrm{Q}^{\mathrm{VAL}-\mathrm{NOC}}$ are the average number of points signalled as out-of-control for the validation of abnormal and NOC batches, respectively, for the squared residuals statistic. $\mathrm{T}^{\mathrm{VAL}-\mathrm{ABN}}$ and $\mathrm{T}^{\mathrm{VAL}-\mathrm{NOC}}$ mean the same but for the Hotelling's $\mathrm{T}^{2}$ statistic. These parameters were obtained by calibrating PCA models under the appropriate conditions $(P C, W S$ ) with the calibration set followed by the projection of the validation set. The $\alpha$ coefficient was introduced to weight the importance of each statistic (here $\alpha=0.75$ weighting preferentially the $\mathrm{Q}$ residuals statistic). The objective was to select the combination $(P C, W S)$ that yielded the maximum value for the $D$ statistic. The results obtained by performing all combinations of WS (1-24) and PC (1-8) were plotted as a contour map (Fig. 4). The contour map showed that models with WS $>20$ and considering up to 3 PC's may be adequate. However, it means that under these circumstances, one may need to wait for almost the entire roasting process to be completed to have a result. In other words, setting WS to $20 \mathrm{~min}$ means that the coffee roasters experts need to wait $20 \mathrm{~min}$ before 


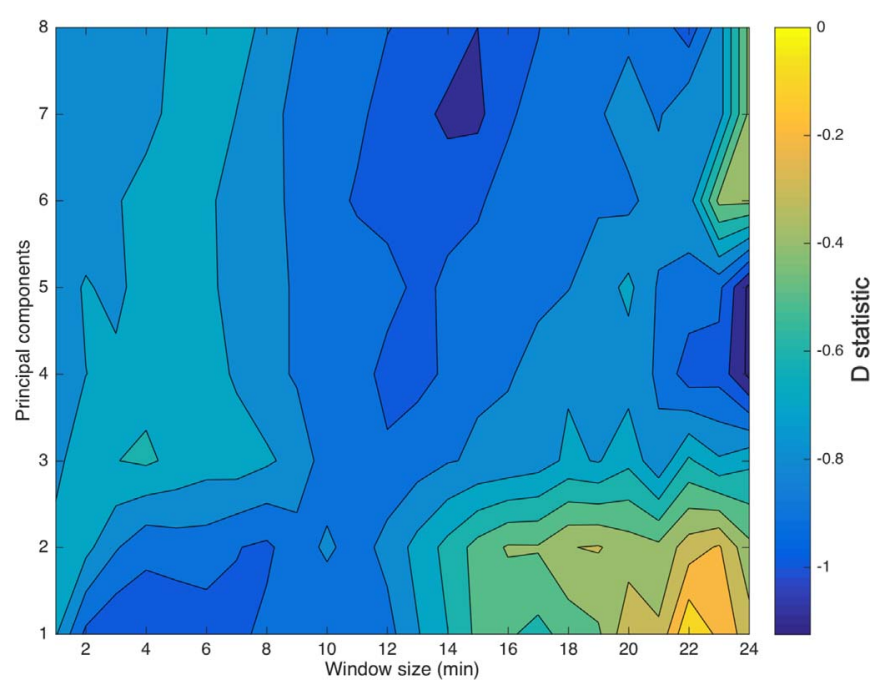

Fig. 4. Contour map of the $D$ parameter obtained from validation batches considering models with different window sizes (WS) and number of principal components (PC) restricted to the $6120-4961 \mathrm{~cm}^{-1}$ spectral region.

having a result. This might be too late if corrective actions are to be implemented. On the other side of the contour map, near WS $=4 \mathrm{~min}$ and $\mathrm{PC}=3$, the $D$ statistic is significantly higher when compared to neighbouring values. Using models with a WS $=4 \mathrm{~min}$, yields a first result after $4 \mathrm{~min}$. Therefore, the combination of $\mathrm{WS}=4 \mathrm{~min}$ and $\mathrm{PC}=$ 3 was adopted. Note that because the WS $=4 \mathrm{~min}$, a total of $21 \mathrm{PCA}$ models were built to cover the entire process time range (spaced of 1 min). Particularly, the first PCA model encompasses the spectra obtained over the initial four minutes of the roasting process. The second PCA model encompasses spectra from 2 to $5 \mathrm{~min}$ and so on. Therefore, setting this model, a coffee roaster expert waits four minutes until a first result regarding the process compliance.

3.3.3. Analysis of the MSPC methodology using the sliding window strategy

To illustrate the results obtained for one model using WS $=4 \mathrm{~min}$ and $\mathrm{PC}=3$, the model encompassing the time section between 21 and 24 min was selected. The score plot obtained for this model (Fig. 5) with overlaid validation and testing batches, showed that the scores for

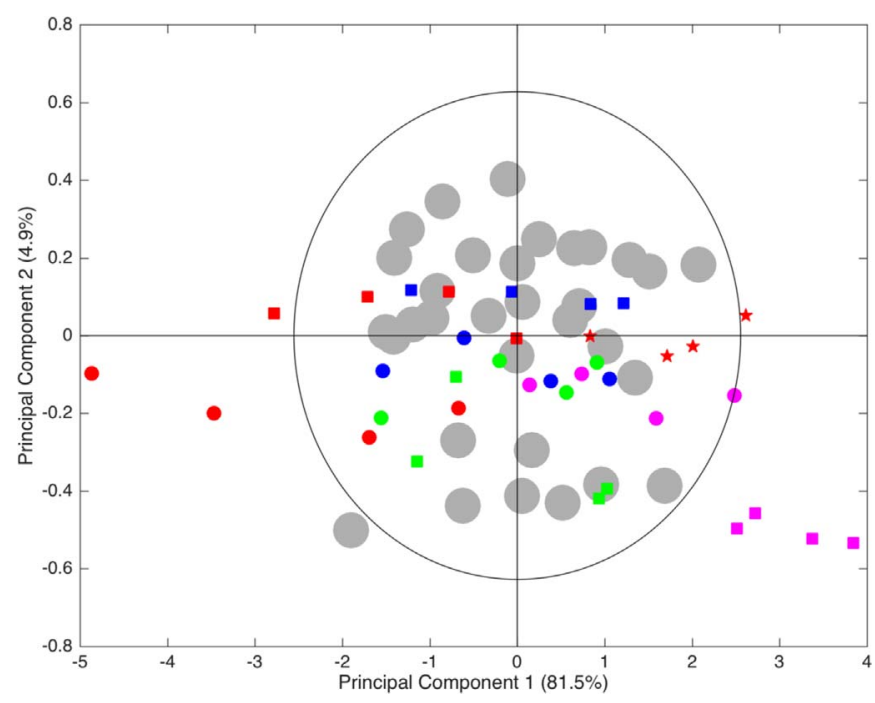

Fig. 5. Score plot for the PCA model encompassing spectra from 21 to $24 \mathrm{~min}$ (WS = $4 \mathrm{~min}$ ), spectral range $6120-4961 \mathrm{~cm}^{-1}$ and 3 PC (only components 1 and 2 are shown) built from calibration batches $(\odot 3,6,7,8,9,10,11,14)$. Validation $(\odot 2, \square 5, \bullet 17$,

$19, \uparrow 20)$ and test $(\square 1, \bigcirc 4, \bigcirc 16, \square 18)$ batches were projected. The boundary defines the $95 \%$ confidence limit considering normally distributed scores. the calibration batches (grey circles) are approximately normally distributed and therefore the confidence limits for the bivariate distribution are more prone to be used effectively. All projected NOC validation/testing batches are within the confidence limit defined by the calibration scores. On the contrary, most of the validation/testing abnormal batches are outside the confidence limit (or at least part of these batches is outside).

The MSPC strategy relies not only in the scores but essentially in the Hotelling's $\mathrm{T}^{2}$ (ultimately defined by the scores and captured variance of each score) and $\mathrm{Q}$ residuals statistics. To highlight the performance of the suggested strategy considering models with WS $=4 \mathrm{~min}$ and 3 PC's, the corresponding Hotelling's $\mathrm{T}^{2}$ and $\mathrm{Q}$ residuals statistics were built. Note that for each batch, there are only 21 scores represented in the Hotelling's and Q residual statistics because WS $=4 \mathrm{~min}$. These 21 scores result from 21 PCA models using 4 spectra each. The corresponding MSPC control charts are shown in Figs. 6 and 7. The MSPC control charts showing calibration and validation batches provide the raw results used by statistic $D$ (Fig. 6). In the normalized Hotelling's $\mathrm{T}^{2}$ chart, the NOC batches used in the calibration and validation process were below the control limit, except one in B6. Regarding the scores of the batches with imposed disturbances: B17 presented higher values than the control limit since the middle of the roasting process; B19 has values above the control limit mainly in the beginning of the roasting process; batch B20 got only one value above the control limit. These results reveal that this methodology is able of detecting batches that are being operated using a higher power (B17) at the middle of the roasting process and using a lower amount of green coffee beans (B19) at the beginning of the roasting process, respectively. Regarding batch B20, this methodology presented some difficulties to identify a roasting process using a higher amount of green coffee beans.

In the normalized $Q$ residuals chart, just a few values of the NOC batches in the calibration and validation set were higher than the control limit. The batches with imposed disturbances (B17 and B19) presented all values above the control limit excluding two in the beginning and three at the middle of the roasting process, respectively; batch $\mathrm{B} 20$ showed some values higher than the control limit mainly in the beginning of the roasting process. The results obtained in this chart are similar to the ones obtained in the normalized Hotelling's $\mathrm{T}^{2}$ chart.

The MSPC control charts of the testing set are shown in Fig. 7. In both normalized Hotelling's $\mathrm{T}^{2}$ and $\mathrm{Q}$ residuals charts, the statistics' values of NOC batches are below the control limit. The same cannot be referred for the batches with imposed disturbances (batches B16 and batch B18). Regarding batch B16, several values were above the confidence limits in both charts. This batch used a different proportion of Arabica and Robusta green coffee beans and it was clearly identified in both charts at the beginning of the roasting process. Batch 18 (B18) presented only the last two time points higher than the confidence limit in the Hotelling's $\mathrm{T}^{2}$ chart but only three points below the confidence limit in the $\mathrm{Q}$ residuals chart. A lower roasting power was used in batch B18 and although not easily identified through the Hotelling's $\mathrm{T}^{2}$ chart, it could be identified in the $\mathrm{Q}$ residual chart. Therefore, the projection of the unseen testing batches (Fig. 7) revealed that this methodology was able not only to signal abnormal batches but also to prevent false alarms for NOC batches. Concerning sensitivity and specificity of the developed models, it is not possible to calculate both parameters. There are situations where these disturbances theoretically should be detected immediately as for the case of batch 16 since different raw material composition was assayed. Indeed MSPC charts detect the disturbance from the beginning as expected. But for other cases (e.g. different roasting powers and/or masses of beans used), the system cannot immediately identify the disturbances because the differences between the chemical/physical parameters of nominal batches and non-nominal batches could only be achieved as the roasting process evolves. It is expected that as the roasting process evolves the deviations become more and more evident, but there is no knowledge about when these deviations should be detected. So, not having this reference, the estimation of sensitivity and specificity is not feasible. 

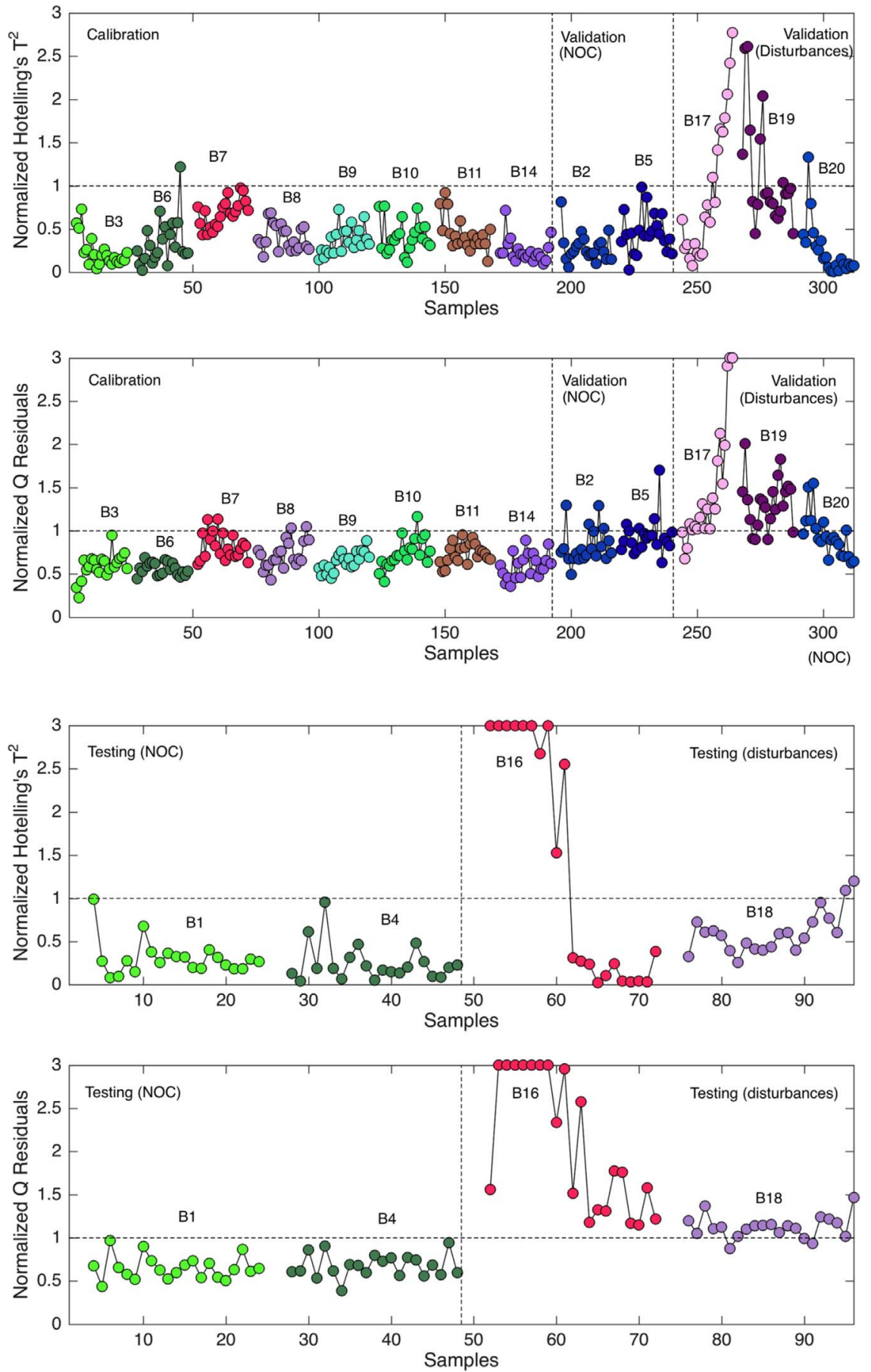

Fig. 6. Normalized Hotelling's $T^{2}$ and $Q$ residuals control charts encompassing the calibration (B3, B6-B11, B14) and validation batches (B2, B5, B17, B19, B20) for models based on WS $=4 \mathrm{~min}$, $\mathrm{PC}=3$ and spectral range $6120-4961 \mathrm{~cm}^{-1}$
Fig. 7. Normalized Hotelling's $T^{2}$ and $Q$ residuals control charts encompassing the testing batches (B1, B4, B16, B18) for models based on WS $=4 \mathrm{~min}, \mathrm{PC}=3$ and spectral range $6120-4961 \mathrm{~cm}^{-1}$ (note: values higher than 3 were set to 3 ).

\section{Conclusions}

For the first time, NIR spectroscopy coupled with MSPC using PCA based models was used to effectively monitor in real-time the coffee roasting process using a conventional roaster. The proposed strategy resourced on a time evolving window to generate sequential models that can better capture the process dynamics by restricting the focus to specific time regions over the roasting process. The suitability of this strategy was demonstrated on a set of roasting batches especially designed. Batches where disturbances were imposed (different roasting conditions and coffee species and origins), were used to optimize the MSPC methodology (validation set) and to test its performance under unseen situations (test set). The optimised methodology, minimizing the number of false positives (specificity) and false negatives (sensitivity), resulted on models encompassing 4 process minutes (meaning that a first result can be obtained after 4 min of process time). The different simulated disturbances could be detected and the time region where the deviation has been observed was compatible with the type of disturbance imposed. Although the obtained results were very satisfactory and promising, further studies including a higher number of non-nominal batches are needed to attest the robustness of this technique. This type of statistical monitoring, combined with a methodology for real-time monitoring (NIR spectroscopy) is a step forward toward a deeper control over roasting processes. In this way, it is possible to decrease the operation costs by avoiding production of faulty roasted coffee batches. Using in-situ real-time NIR spectroscopy for early fault detection and to estimate several parameters (such as sugar, caffeine, colour, antioxidant capacity among others), makes this 
methodology undoubtedly useful as a support tool for roasted coffee production and/or product development as well as coffee roasters experts' decisions.

\section{Acknowledgements}

Tiago A. Catelani is grateful to CAPES for a scholarship (PDSE \#99999.000655/2015-05) and to CNPq. R.N.M.J. Páscoa acknowledges the grant SFRH/BPD/81384/2011. This work also received financial support from the European Union (FEDER funds POCI/01/0145/ FEDER/007265) and National Funds (FCT/MEC, Fundação para a Ciência e Tecnologia and Ministério da Educação e Ciência) under the Partnership Agreement PT2020 UID/QUI/50006/2013 and iMed.ULisboa grant UID/DTP/04138/2013.

\section{References}

[1] ICO, International Coffee Organization; 2016. 〈http://www.ico.org $\rangle$. (Accessed in 01 June 2016).

[2] J.R. Santos, O. Viegas, R.N. Páscoa, I.M. Ferreira, A.O. Rangel, J.A. Lopes, In-line monitoring of the coffee roasting process with near infrared spectroscopy: measurement of sucrose and colour, Food Chem. 208 (2016) 103-110.

[3] R. Eggers, A. Pietsch, Technology I: Roasting, Coffee, Blackwell Science Ltd, 2008, pp. 90-107.

[4] WCT, World Coffee Trade - An Overview The Coffee Exporter's guide, International Trade Centre (ITC) Publication, Geneva, 2011, p. 20.

[5] M.C. Sarraguca, P.R.S. Ribeiro, A.O. Dos Santos, J.A. Lopes, Batch statistical process monitoring approach to a cocrystallization process, J. Pharm. Sci. 104 (2015) 4099-4108.

[6] A.S.S. Pinto, S.C. Pereira, M.P.A. Ribeiro, C.S. Farinas, Monitoring of the cellulosic ethanol fermentation process by near-infrared spectroscopy, Bioresour. Technol. 203 (2016) 334-340.

[7] J.R. Santos, M. Lopo, A. Rangel, J.A. Lopes, Exploiting near infrared spectroscopy as an analytical tool for on-line monitoring of acidity during coffee roasting, Food Control 60 (2016) 408-415.

[8] M.R. Ruosi, C. Cordero, C. Cagliero, P. Rubiolo, C. Bicchi, B. Sgorbini, E. Liberto, A further tool to monitor the coffee roasting process: aroma composition and chemical indices, J. Agric. Food Chem. 60 (2012) 11283-11291.

[9] P.S. Wilson, Coffee roasting acoustics, J. Acoust. Soc. Am. 135 (2014) EL265-EL269.

[10] T. Kourti, Multivariate dynamic data modeling for analysis and statistical proces control of batch processes, start-ups and grade transitions, J. Chemometr. 17 (2003) 93-109.

[11] T. Kourti, Application of latent variable methods to process control and multivariate statistical process control in industry, Int. J. Adapt. Control Signal Process. 19 (2005) 213-246.

[12] W.L. Li, H.F. Han, Z.W. Cheng, Y. Zhang, S.Y. Liu, H.B. Qu, A feasibility research on the monitoring of traditional Chinese medicine production process using NIR-based multivariate process trajectories, Sens. Actuator B-Chem. 231 (2016) 313-323.
[13] S. Verdu, F. Vasquez, R. Grau, E. Ivorra, A.J. Sanchez, J.M. Barat, Detection of adulterations with different grains in wheat products based on the hyperspectral image technique: the specific cases of flour and bread, Food Control 62 (2016) 373-380.

[14] R. Hertz-Schunemann, R. Dorfner, C. Yeretzian, T. Streibel, R. Zimmermann, Online process monitoring of coffee roasting by resonant laser ionisation time-of-flight mass spectrometry: bridging the gap from industrial batch roasting to flavour formation inside an individual coffee bean, J. Mass Spectrom. 48 (2013) 1253-1265

[15] A.N. Gloess, A. Vietri, F. Wieland, S. Smrke, B. Schonbachler, J.A.S. Lopez, S. Petrozzi, S. Bongers, T. Koziorowski, C. Yeretzian, Evidence of different flavour formation dynamics by roasting coffee from different origins: on-line analysis with PTR-ToF-MS, Int. J. Mass Spectrom. 365 (2014) 324-337.

[16] F. Wieland, A.N. Gloess, M. Keller, A. Wetzel, S. Schenker, C. Yeretzian, Online monitoring of coffee roasting by proton transfer reaction time-of-flight mass spectrometry (PTR-ToF-MS): towards a real-time process control for a consistent roast profile, Anal. Bioanal. Chem. 402 (2012) 2531-2543.

[17] B.A. Goodman, E.C. Pascual, C. Yeretzian, Real time monitoring of free radical processes during the roasting of coffee beans using electron paramagnetic resonance spectroscopy, Food Chem. 125 (2011) 248-254.

[18] T. Catelani, R. Pascoa, J.R. Santos, L. Pezza, H. Pezza, J. Lima, J.A. Lopes, A noninvasive real-time methodology for the quantification of antioxidant properties in coffee during the roasting process based on near-infrared spectroscopy, food and bioprocess, Technology 10 (2017) 630-638.

[19] S. Wold, N. Kettaneh, H. Friden, A. Holmberg, Modelling and diagnostics of batch processes and analogous kinetic experiments, Chemom. Intell. Lab. Syst. 44 (1998) 331-340.

[20] I.T. Jolliffe, Principal Component Analysis, Springer-Verlag, New York, 2002.

[21] J.A. Lopes, J.C. Menezes, Trilinear models for batch MSPC: application to an industrial batch pharmaceutical process, in: J. Grievink, J. VanSchijndel (Eds.), European Symposium on Computer Aided Process Engineering - 12, Elsevier Science Bv, Amsterdam, 2002, pp. 709-714.

[22] D.A.O. Moraes, F.L.P. Oliveira, L.H. Duczmal, F.R.B. Cruz, Comparing the inertial effect of MEWMA and multivariate sliding window schemes with confidence contro charts, Int. J. Adv. Manuf. Technol. 84 (2016) 1457-1470.

[23] P. Nomikos, J.F. Macgregor, Monitoring batch processes using multiway principal component analysis, AIChE J. 40 (1994) 1361-1375.

[24] P. Nomikos, J.F. Macgregor, Multivariate SPC charts for monitoring batch processes, Technometrics 37 (1995) 41-59.

[25] F. Wei, M. Tanokura, Chapter 17 organic compounds in green coffee beans, in: V.R. Preedy (Ed.), Coffee Health and Disease Prevention, Elsevier, London, UK, 2015.

[26] I. Esteban-Diez, J.M. Gonzalez-Saiz, C. Pizarro, Prediction of sensory properties of espresso from roasted coffee samples by near-infrared spectroscopy, Anal. Chim. Acta 525 (2004) 171-182.

[27] A.P. Craig, A.S. Franca, L.S. Oliveira, J. Irudayaraj, K. Ileleji, Application of elastic net and infrared spectroscopy in the discrimination between defective and nondefective roasted coffees, Talanta 128 (2014) 393-400.

[28] J.S. Ribeiro, M.M.C. Ferreira, T.J.G. Salva, Chemometric models for the quantitative descriptive sensory analysis of Arabica coffee beverages using near infrared spectroscopy, Talanta 83 (2011) 1352-1358.

[29] C. Pizarro, I. Esteban-Diez, J.M. Gonzalez-Saiz, M. Forina, Use of near-infrared spectroscopy and feature selection techniques for predicting the caffeine content and roasting color in roasted coffees, J. Agric. Food Chem. 55 (2007) 7477-7488. 